\title{
Fuel retention in carbon materials under ITER-relevant mixed species plasma conditions
}

\author{
A Kreter ${ }^{1}$, M J Baldwin ${ }^{2}$, R P Doerner ${ }^{2}$, D Nishijima ${ }^{2}$, P Petersson ${ }^{3}$, \\ A Pospieszczyk ${ }^{1}, M_{\text {Rubel }}^{4}$ and K Umstadter ${ }^{2}$ \\ ${ }^{1}$ Institute for Energy Research-Plasma Physics, Forschungszentrum Juelich, \\ Association EURATOM-FZJ, Trilateral Euregio Cluster, Germany \\ ${ }^{2}$ Center for Energy Research, University of California at San Diego, 9500 Gilman Drive, \\ La Jolla, CA 92093-0417, USA \\ ${ }^{3}$ Tandem Laboratory, Uppsala University, Association EURATOM-VR, Box 529, \\ 75120 Uppsala, Sweden \\ ${ }^{4}$ Alfvén Laboratory, Royal Institute of Technology (KTH), Association EURATOM-VR, \\ 10044 Stockholm, Sweden \\ E-mail: a.kreter@fz-juelich.de
}

Received 28 October 2009

Accepted for publication 5 November 2009

Published 30 December 2009

Online at stacks.iop.org/PhysScr/T138/014012

\begin{abstract}
Samples of CFC NB41 and fine-grain graphite ATJ have been exposed to PISCES plasmas containing (i) pure deuterium, (ii) deuterium and beryllium, (iii) deuterium, beryllium and helium, and (iv) deuterium, beryllium and argon. Thermal desorption spectrometry (TDS) and nuclear reaction analysis (NRA) have been used to measure the amount and distribution of retained deuterium in the samples. For the case of pure deuterium plasma, parametric studies of deuterium retention in NB41 have been done with variations of the incident deuterium fluence $\left(\Phi=1 \times 10^{25}-5 \times 10^{26} \mathrm{~m}^{-2}\right)$, ion energy $\left(E_{\mathrm{i}}=20-120 \mathrm{eV}\right)$ and sample surface temperature $\left(T_{\mathrm{s}}=370-820 \mathrm{~K}\right)$. It has been found, that for $T_{\mathrm{s}}=470 \mathrm{~K}$ the retention scales as $\Phi^{0.35}$. For $T_{\mathrm{s}}=820 \mathrm{~K}$ the retention saturates at a level of $\sim 10^{21} \mathrm{D} \mathrm{m}^{-2}$. The retention increases with $E_{\mathrm{i}}$ and drops with higher $T_{\mathrm{s}}$. At $T_{\mathrm{s}}=720 \mathrm{~K}$, the beryllium seeding results in a building of a protective beryllium carbide layer, which appears to prevent the in-bulk diffusion of deuterium, thus reducing the retention. Admixture of $\mathrm{Ar}$ and, in the case of low $E_{\mathrm{i}}$, He leads to a significant reduction of the retention.
\end{abstract}

PACS numbers: 28.52.Fa, 52.40.Hf, 52.55.-s

(Some figures in this article are in colour only in the electronic version.)

\section{Introduction}

The first generation of the ITER divertor will employ carbon fibre composite (CFC) for the strike point areas and tungsten for the baffles [1]. CFC NB41 produced by Snecma, which has superior thermo-mechanical properties with respect to other CFC grades, is intended to be used in International Thermonuclear Experimental Reaction (ITER) [2]. While an extensive database is available on the retention properties of different carbon-based materials [3-10], the corresponding data for the novel NB41 material are yet to be collected.
The current knowledge on the hydrogen retention mechanisms responsible for the most of retention in carbon-based materials can be summarized as follows: (i) the incident hydrogen ions penetrate into the surface layer according to their incident energies $(\sim 10$ to $\sim 100 \mathrm{~nm}$ for the energy range of $\sim 100 \mathrm{eV}$ to several $\mathrm{keV}$, typical for the ion-beam devices). The so-called saturated layer at a level of a few $10^{20} \mathrm{~m}^{-2}$ is typically created after a dose of $\sim 10^{21} \mathrm{~m}^{-2}$ by a balance between the incident ions and the recycling atoms. (ii) If the bulk material is porous, hydrogen can diffuse along the surface of interconnected pores deeper into the bulk. This 
is the case for most of CFC and fine-grain graphite (FGG) materials with their typical porosity of $10-20 \%$. At moderate surface temperatures ( $T_{\mathrm{s}}$ between room temperature and a few hundreds K), this deep in-bulk retention scales with the square root of the fluence even for the highest doses obtained so far in the experiments.

The aim of this work was to investigate NB41 with respect to the in-bulk fuel retention and compare it with other carbon materials for conditions relevant to the ITER divertor plasma. The distinct features in the ITER divertor will be a high deuterium ion flux (up to $\Gamma_{\mathrm{D}} \sim 10^{24} \mathrm{~m}^{-2} \mathrm{~s}^{-1}$ ) and fluence ( $\Phi>10^{26} \mathrm{~m}^{-2}$ at the strike point for single ITER pulse), a rather low incident ion energy of $E_{\mathrm{i}} \sim 10 \mathrm{eV}$ and surface temperatures in the range of $T_{\mathrm{s}}=500-1000 \mathrm{~K}$. The divertor target will be subjected to mixed species fluxes containing hydrogenic isotopes $(\mathrm{H} / \mathrm{D} / \mathrm{T})$, beryllium eroded from the main wall, helium produced in D-T reactions and argon (or neon) applied as an external radiator to distribute power from the divertor plasma.

The experimental procedure in the PISCES-A and PISCES-B linear plasma devices and the covered range of exposure parameters are given in section 2. The behaviour of the fuel retention in NB41 exposed to pure deuterium plasma is described to the full extent in [11]. Here, these results are summarized in section 3. A broader insight is given for the case of plasmas with the $\mathrm{Be}, \mathrm{He}$ and Ar impurity seeding in section 4. The implications for ITER are discussed in section 5 .

\section{Experimental procedure and post-mortem analyses}

The results presented here were obtained in the PISCES-A and PISCES-B linear plasma devices [12]. Both devices are similar in their parameters, the main difference is that PISCES-B is capable of producing Be containing plasmas and handling Be contaminated samples. Naturally, the operational domain of the linear device do not fully cover the plasma parameters in ITER. Typical plasma parameters in the experiments presented here were an electron density of $n_{\mathrm{e}}=$ $(2-3) \times 10^{18} \mathrm{~m}^{-3}$, an electron temperature of $T_{\mathrm{e}}=7-15 \mathrm{eV}$, resulting in an incident ion flux at the sample of $\Gamma_{\mathrm{D}}=(3-6) \times$ $10^{22} \mathrm{~m}^{-2} \mathrm{~s}^{-1}$. However, the parameters important with respect to the retention studies can be reproduced in the devices. So, the incident ion energy $E_{\mathrm{i}}$ was varied between 20 and $120 \mathrm{eV}$ by choosing an appropriate target bias potential, the sample temperature $T_{\mathrm{s}}$ between $370 \mathrm{~K}$ (representing the 'cold' ITER wall) and $820 \mathrm{~K}$ ( $T_{\mathrm{s}}$ at the strike point) by adjusting the plasma parameters and the sample cooling efficiency, and the incident deuterium fluence between $1 \times 10^{25} \mathrm{~m}^{-2}$ and $5 \times 10^{26} \mathrm{~m}^{-2}$ (the latter value representing one ITER pulse at the strike point) by extending the steady-state plasma exposure from a few minutes to several hours. Be, He and Ar were seeded into the plasma in a controlled manner to simulate the mixed plasma conditions in ITER. The impurity content in the plasma is measured by the optical spectroscopy [13].

After plasma exposures, the samples were analysed for the amount of retained deuterium by the thermal desorption spectrometry (TDS) with a typical rate of the temperature

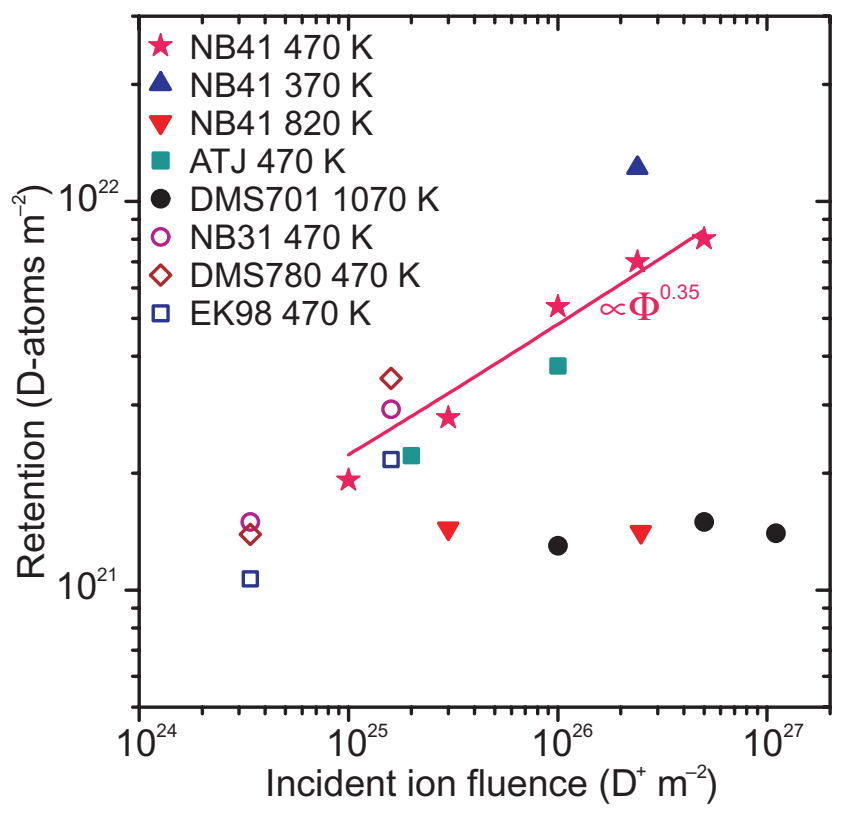

Figure 1. Total amount of retained deuterium as function of the incident fluence. The data from PISCES (filled symbols) for CFC NB41, FGG ATJ and CFC DMS701 [14] and from TEXTOR (open symbols) for CFC NB31, CFC DMS780 and FGG EK98 [15] are shown for different exposure temperatures according to the legend. All samples were exposed with an incident ion energy of $100-150 \mathrm{eV}$, except for CFC DMS701 $\left(E_{\mathrm{i}} \approx 30 \mathrm{eV}\right)$. The line represents the fit of the CFC NB41 data for $T_{\mathrm{s}}=470 \mathrm{~K}$ by function $\propto \Phi^{\gamma}$, resulting in $\gamma=0.35$.

increase of $0.5 \mathrm{~K} \mathrm{~s}^{-1}$. The nuclear reaction analysis (NRA) with ${ }^{3} \mathrm{He}^{+} 2 \mathrm{MeV}$ beam was applied to measure the depth distribution of deuterium in the surface layer corresponding to the penetration depth of the beam.

\section{Retention in CFC NB41 exposed to pure deuterium plasma}

Parametric studies of the fuel retention in CFC NB41 have been performed [11]. Figure 1 summarizes the total amount of deuterium retained in samples exposed to different doses at surface temperatures of 370, 470 and $820 \mathrm{~K}$. Data for other carbon materials from previous experiments in PISCES [14] and the TEXTOR tokamak [15] are shown for comparison.

For $T_{\mathrm{s}}=470 \mathrm{~K}$, the retention in NB41 scales with the fluence as $\Phi^{0.35}$, close to the square-root dependence characteristic for the in-bulk retention in other CFC materials [10]. The good agreement in absolute values between different CFC materials (NB41, former ITER-grade NB31, JET type CFC DMS780 and Tore Supra type CFC N11 [16]) underlines the similarity of the retention mechanisms in these materials. The retention in FGG EK98 exposed in TEXTOR and ATJ exposed in PISCES is on average $\sim 30 \%$ lower than in CFCs exposed at the same conditions, but exhibits a similar dependence on the fluence.

The retention in NB41 is lower when the sample is exposed at higher temperature. This dependence on $T_{\mathrm{S}}$ is in agreement with previous studies [7, 16] and is due to the presence of additional traps for hydrogen in the carbon material at low $T_{\mathrm{s}}$ and higher population in available traps [11]. The apparent absence of traps, which are largely 
responsible for the in-bulk retention, at temperatures higher than $\approx 800 \mathrm{~K}$ leads to a saturation of retention at a level of $\sim 10^{21} \mathrm{~m}^{-2}$ for NB41 and ASDEX Upgrade grade CFC DMS701 [14].

Among other results from the parametric studies performed in [11], one should mention the increase of the retention in NB41 by a factor of $\approx 2$ when changing the incident energy from 20 to $120 \mathrm{eV}$. This can be attributed to a higher level of saturation of the implantation layer for higher energies (cf [3]) and, correspondingly, a higher diffusive hydrogen flux into the bulk.

From the cross-machine comparison of retention studies [11] a dependence of the retention on the incident particle flux can be deduced. In the case of lower fluxes, e.g. typical for the ion-beam devices $\left(\Gamma_{\mathrm{D}} \sim 10^{19} \mathrm{~m}^{-2} \mathrm{~s}^{-1}\right)$ versus plasma devices like PISCES and TEXTOR $\left(\Gamma_{\mathrm{D}} \sim 10^{22}-10^{23} \mathrm{~m}^{-2} \mathrm{~s}^{-1}\right)$, the longer time needed to collect the same dose leads to a higher amount of deuterium diffusing into the bulk.

The D depth profiling by NRA of the exposed NB41 samples showed that the concentration of $\mathrm{D}$ at the surface corresponds to the saturation level for the particular surface temperature. However, D has been found in the bulk clearly deeper than the implantation region.

\section{Influence of impurities on the in-bulk retention}

\subsection{Retention with D-Be mixed species plasma}

The experiments for the influence of the impurities on the bulk retention were done using FGG ATJ samples. Due to similar retention in the CFC and FGG materials found for the pure deuterium plasma conditions (section 3), it can be expected that the effects induced by impurities for FGG ATJ are transferable to the case of CFC materials.

From the previous experiments with Be seeding in PISCES it has been found that at surface temperatures greater than $600 \mathrm{~K}$ Be reacts with the carbon substrate to build a beryllium carbide layer with a typical thickness in steady-state of several $100 \mathrm{~nm}$ [17]. One of the consequences of this mixed material layer is a significant reduction of carbon erosion from the target surface $[18,19]$. To check the influence of the Be carbide layer on the retention, the surface temperature has been chosen to be $720 \mathrm{~K}$, high enough for a rapid development of the Be carbide layer and low enough to avoid the saturation of the retention, which sets in at $\approx 800 \mathrm{~K}$. Reference ATJ samples have been exposed to the pure D plasma for otherwise similar plasma parameters.

The typical scenario of a Be seeding experiment at PISCES-B consists of two stages. At first, prior to the Be injection, the target plasma with parameters required by the particular experiment is established. Then, the shutter of the Be oven is opened to start the Be seeding. The amount of $\mathrm{Be}$ is controlled by the oven temperature and was typically at a Be/D-level of $\sim 0.1 \%$ in the experiments presented here. To make the direct comparison of different experiments possible, the deuterium fluences at both experimental stages are kept reproducible: $0.5 \times 10^{26} \mathrm{~m}^{-2}$ prior to the $\mathrm{Be}$ seeding and $2 \times 10^{26} \mathrm{~m}^{-2}$ in total (the fluence prior to the Be seeding plus the fluence during the seeding).

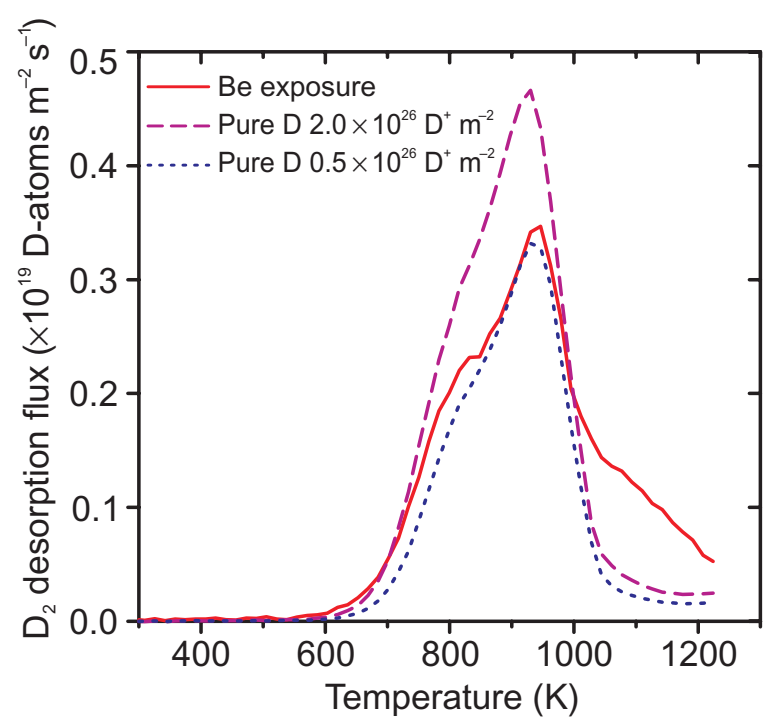

Figure 2. TDS spectra of mass $4\left(\mathrm{D}_{2}\right)$ from ATJ samples exposed to a Be containing plasma (full line), pure D plasma with $\Phi=2 \times 10^{26} \mathrm{~m}^{-2}$ (dashed line) and pure D plasma with $\Phi=0.5 \times 10^{26} \mathrm{~m}^{-2}$ (dotted line). All exposures were done at $T_{\mathrm{s}}=720 \mathrm{~K}$ and $E_{\mathrm{i}}=35 \mathrm{eV}$.

Figure 2 shows the outgassing deuterium flux from a sample exposed to a Be containing plasma. Here, as in all subsequently presented TD spectra of mass 4 , the outgassing flux of deuterium molecules is given in units of D-atoms $\mathrm{m}^{-2} \mathrm{~s}^{-1}$. The thermal desorption peak between 800 and $1000 \mathrm{~K}$, at which the most amount of retained deuterium is released, is significantly reduced in comparison to the reference sample exposed to the same total dose $\left(\Phi=2 \times 10^{26} \mathrm{~m}^{-2}\right)$. Instead, this TDS peak is well reproduced by the reference sample exposed to a fluence corresponding to the first non-beryllium stage of the experiment $\left(\Phi=0.5 \times 10^{26} \mathrm{~m}^{-2}\right)$. These comparisons show that apparently the formation of Be carbide layer prevents further increase of the D retention with fluence. Because of significantly larger thickness of the Be carbide layer with respect to the D implantation layer (several $100 \mathrm{~nm}$ and $\sim 10 \mathrm{~nm}$, respectively), it can effectively block the in-bulk diffusion channel of deuterium. In terms of the total amount of retained deuterium (table 1), the addition of Be reduces the retention in comparison with the pure $\mathrm{D}$ case for the same total fluence $\left(\Phi=2 \times 10^{26} \mathrm{~m}^{-2}\right)$. However, the Be seeding increases the retention with respect to the initial, non-Be stage of the experiment (cf retention in the reference sample exposed to $\Phi=0.5 \times 10^{26} \mathrm{~m}^{-2}$ in table 1 ). This is mainly because of the additional $\mathrm{D}$ trapping in the Be exposed sample corresponding to outgassing temperatures between 1000 and $1200 \mathrm{~K}$. Presumably, these additional trapping sites are associated with Be carbide.

\subsection{Retention with $\mathrm{D}-\mathrm{Be}-\mathrm{He}$ mixed species plasma}

To investigate the influence of $\mathrm{He}$ on the deuterium retention, a similar experimental procedure has been used as in the case of Be seeding. First, a mixed deuterium-helium background plasma is established, before beryllium is seeded. The surface temperature was kept at $720 \mathrm{~K}$. The experiments were done for two different values of the incident ion energy: 35 and $80 \mathrm{eV}$. 
Table 1. Total amounts of deuterium retention in exposed ATJ samples. The surface temperature was kept constant at $720 \mathrm{~K}$ for all exposures.

\begin{tabular}{|c|c|c|c|c|c|c|c|}
\hline $\begin{array}{l}\text { Plasma species } \\
\text { (fractions) }\end{array}$ & $\mathrm{D}$ & $\mathrm{D}$ & $\begin{array}{c}\mathrm{D} / \\
\mathrm{Be}(0.0005)\end{array}$ & $\begin{array}{c}\mathrm{D} / \\
\mathrm{Be}(0.0009) / \\
\mathrm{He}(0.16)\end{array}$ & $\begin{array}{c}\mathrm{D} / \\
\mathrm{Be}(0.0008) / \\
\mathrm{He}(0.4)\end{array}$ & $\begin{array}{c}\mathrm{D} / \\
\mathrm{Be}(0.0023) / \\
\mathrm{Ar}(0.1)\end{array}$ & $\begin{array}{c}\mathrm{D} / \\
\operatorname{Be}(0.006) / \\
\operatorname{Ar}(0.1)\end{array}$ \\
\hline $\begin{array}{l}\text { Incident ion } \\
\text { energy }(\mathrm{eV})\end{array}$ & 35 & 35 & 35 & 35 & 80 & 35 & 120 \\
\hline $\begin{array}{l}\text { D fluence before } \\
\text { Be seeding }\left(\mathrm{m}^{-2}\right)\end{array}$ & N/A & N/A & $0.5 \times 10^{26}$ & $0.4 \times 10^{26}$ & $0.3 \times 10^{26}$ & $0.1 \times 10^{26}$ & $0.1 \times 10^{26}$ \\
\hline $\begin{array}{l}\text { Total D fluence } \\
\left(\mathrm{m}^{-2}\right)\end{array}$ & $0.5 \times 10^{26}$ & $2.0 \times 10^{26}$ & $2.0 \times 10^{26}$ & $1.7 \times 10^{26}$ & $1.2 \times 10^{26}$ & $0.5 \times 10^{26}$ & $0.5 \times 10^{26}$ \\
\hline $\begin{array}{l}\text { Total D retention } \\
\left(\mathrm{m}^{-2}\right)\end{array}$ & $1.6 \times 10^{21}$ & $2.3 \times 10^{21}$ & $1.9 \times 10^{21}$ & $0.5 \times 10^{21}$ & $3.6 \times 10^{21}$ & $0.8 \times 10^{21}$ & $0.4 \times 10^{21}$ \\
\hline
\end{tabular}

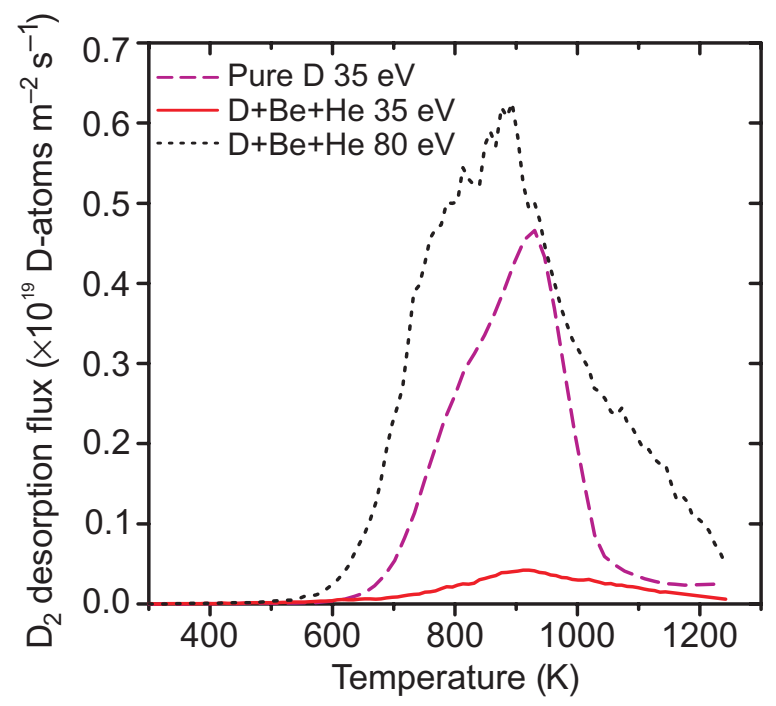

Figure 3. TDS spectra of mass $4\left(\mathrm{D}_{2}\right)$ from ATJ samples exposed to pure D plasma at $E_{\mathrm{i}}=35 \mathrm{eV}$ (dashed line) and mixed D/Be/He plasma at $E_{\mathrm{i}}=35 \mathrm{eV}$ (full line) and at $E_{\mathrm{i}}=80 \mathrm{eV}$ (dotted line). All exposures were done at $T_{\mathrm{s}}=720 \mathrm{~K}$.

In the case of low energy a strong reduction of the deuterium retention has been observed (figure 3 , see also table 1 for total numbers). The retention dropped even in comparison with the $\mathrm{D} / \mathrm{Be}$ case, in which the retention was already reduced in comparison to the pure D plasma (cf sections 4.1). This suggests an effective He-induced detrapping of $\mathrm{D}$, which in the case of the low incident energy is implanted close to the surface and is therefore easier to remove.

Unfortunately, there are no pure deuterium or $\mathrm{D} / \mathrm{Be}$ reference experiments for the case of $E_{\mathrm{i}}=80 \mathrm{eV}$. However, there is no such evident reduction effect on the $\mathrm{D}$ retention by $\mathrm{He}$ as in the case of the low incident energy, despite higher He concentration during the exposure (table 1). Presumably, at the higher energy the deeper implanted deuterium cannot be effectively desorbed by He. The retention is higher than in the available reference with pure D plasma and $E_{\mathrm{i}}=35 \mathrm{eV}$, which can be attributed to the difference in the incident energy (cf section 3). Investigations in PISCES showed, that the retention in a Be target is reduced significantly if exposed to the $\mathrm{D} / \mathrm{He}$ mixed plasma in comparison to the pure $\mathrm{D}$ plasma. In the particular pair of exposures shown in figure 4 , the

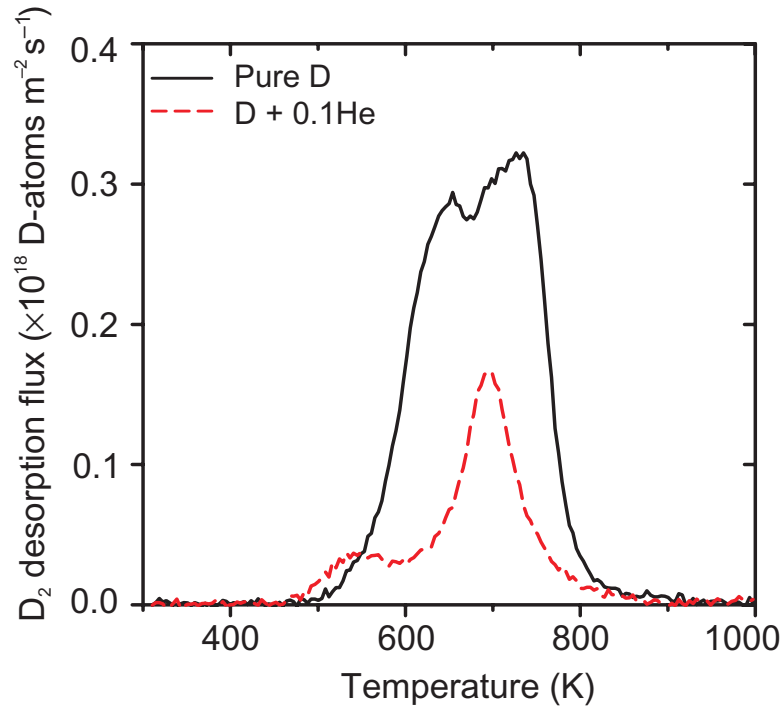

Figure 4. TDS spectra of mass $4\left(\mathrm{D}_{2}\right)$ from beryllium samples exposed to pure D plasma (full line) and mixed D/He (10\% of He) plasma (dashed line). Both exposures were done at $T_{\mathrm{s}}=470 \mathrm{~K}$ and $E_{\mathrm{i}}=30 \mathrm{eV}$ with $\Phi=3 \times 10^{25} \mathrm{~m}^{-2}$.

addition of $10 \% \mathrm{He}$ to the deuterium plasma reduces the total $\mathrm{D}$ retention in $\mathrm{Be}$ from $2.3 \times 10^{20} \mathrm{~m}^{-2}$ to $0.8 \times 10^{20} \mathrm{~m}^{-2}$ for otherwise similar exposure conditions $\left(\Phi=3 \times 10^{25} \mathrm{~m}^{-2}\right.$, $T_{\mathrm{s}}=470 \mathrm{~K}$ and $\left.E_{\mathrm{i}}=30 \mathrm{eV}\right)$. In the case of D/Be/He mixed species, the effect of $\mathrm{He}$ on the retention in Be deposited on the target during the exposure can also play a role in the observed reduction of the retention. Because of a larger amount of Be typically deposited on the sample in steady state at lower incident energies [19], the effect of Be on the retention and its reduction by $\mathrm{He}$ would be stronger than at high energies, as observed here.

\subsection{Retention with $D-B e-A r$ mixed species plasma}

In the experiments with argon, the following experimental scenario was used. After a short initial phase with the mixed D/Ar plasma, corresponding to a D fluence of $\Phi \approx 1 \times 10^{25} \mathrm{~m}^{-2}$, Be was added to the plasma. The total deuterium fluence was $\Phi \approx 5 \times 10^{25} \mathrm{~m}^{-2}$. The experiments were performed at two values of the incident ion energy, 35 and $120 \mathrm{eV}$, with a surface temperature of $720 \mathrm{~K}$. Figure 5 


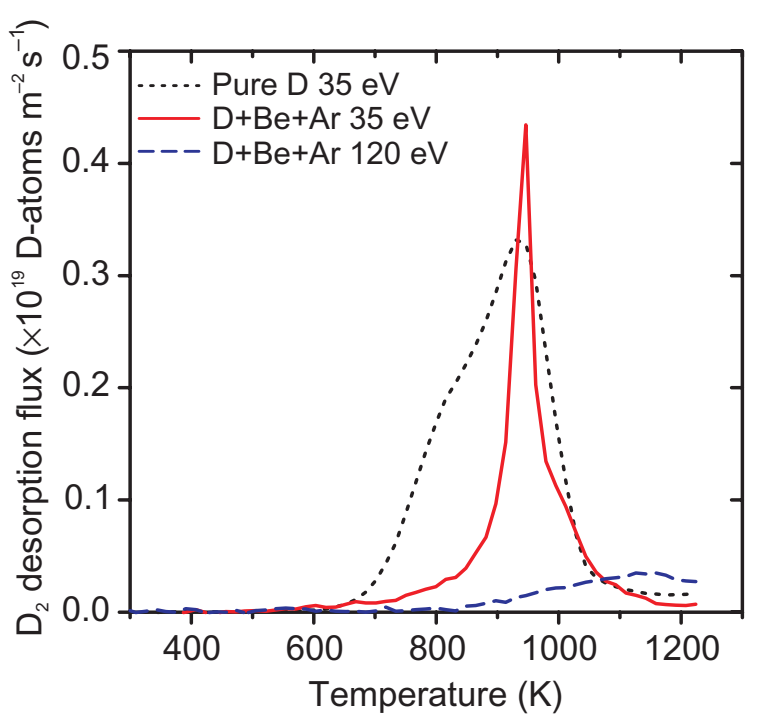

Figure 5. TDS spectra of mass $4\left(\mathrm{D}_{2}\right)$ from ATJ samples exposed to pure D plasma at $E_{\mathrm{i}}=35 \mathrm{eV}$ (dotted line) and mixed D/Be/Ar plasma at $E_{\mathrm{i}}=35 \mathrm{eV}$ (full line) and at $E_{\mathrm{i}}=120 \mathrm{eV}$ (dashed line). All exposures were done at $T_{\mathrm{s}}=720 \mathrm{~K}$.

shows the TDS spectra of these samples along with the reference sample of pure D plasma at $E_{\mathrm{i}}=35 \mathrm{eV}$ and $\Phi=$ $5 \times 10^{25} \mathrm{~m}^{-2}$. Though the direct comparison to the He case is difficult due to the significantly lower exposure dose, it appears that, unlike the case of $\mathrm{He}$, the ion-induced $\mathrm{D}$ detrapping by Ar is more effective at the high ion energy. At the low energy, the $\mathrm{D}_{2}$ release is reduced to a sharp peak at $930 \mathrm{~K}$, underlining the changes in the retention mechanism in the presence of Ar. The total amount of retention is reduced in both low and high energy cases in comparison to the pure D plasma reference (table 1).

\section{Summary and discussion}

The novel ITER-grade CFC NB41 has been characterized with respect to the in-bulk deuterium retention. It has been found, that retention in NB41 obeys the similar laws as other CFC materials. Therefore, predictions for ITER, which were based on the tests of other CFC materials, are generally still valid. However, to describe the retention in a CFC material correctly, the dependences on the incident fluence, flux, ion energy and surface temperature have to be taken into account. As an example, the retention in CFC estimated in [20] has been based on the measurements with the low flux ion beams. Not taking into account the dependence of the flux has resulted in a significant overestimation of the in-bulk CFC retention.

Investigations of the effects of plasma impurities Be, $\mathrm{He}$ and $\mathrm{Ar}$ on the retention have revealed several potentially positive aspects for ITER. The formation of a Be carbide layer on the graphitic surface leads to a blocking of deuterium diffusion into the bulk. Addition of $\mathrm{He}$ and $\mathrm{Ar}$ reduces the retention for certain exposure conditions. He appears to prevent D retention especially effective at the ITER relevant low impact energies. Although the origin of the effect of $\mathrm{He}$ and Ar can generally be reduced to the ion-induced detrapping of deuterium, the differences in applicable conditions for $\mathrm{He}$ and Ar reveal the specifics of the retention mechanism in each of the cases. Further experimental investigations and modelling are necessary to unveil these specific properties. One of the questions to clarify is whether the ion-induced detrapping by impurities would lead to a significant decrease of the hydrogen fraction in carbon co-deposits, which still remain the main concern with respect to the tritium retention in ITER. It has been identified in [21], that introducing Ar in the $\mathrm{D}$ plasma reduces the $\mathrm{D}$ fraction in tungsten co-deposits in the remote area in PISCES.

\section{Acknowledgments}

We thank the engineering staff of the PISCES lab at UCSD, in particular Leo Chousal, Rollie Hernandez, Tyler Lynch and Ray Seraydarian, for their excellent support in the preparation and the execution of the experiments. This work was supported by the EU-US bilateral collaboration on mixed-material plasma-surface interaction effects for ITER.

Published under licence from EURATOM.

\section{References}

[1] Progress in the ITER Physics Basis 2007 Nucl. Fusion $47 \mathrm{~S} 1$

[2] Peacock A T et al 2007 Phys. Scr. T 12823

[3] Staudenmaier G et al 1979 J. Nucl. Mater. 84149

[4] Langley R A 1987 J. Vac. Sci. Technol. A 52205

[5] Wilson K L and Hsu W L 1987 J. Nucl. Mater. 145-147 121

[6] Möller W 1989 J. Nucl. Mater. 162-164 138

[7] Causey R A 1989 J. Nucl. Mater. 162-164 151

[8] Balden M et al 2003 Phys. Scr. T 10338

[9] Atsumi H 2003 J. Nucl. Mater. 313-316 543

[10] Alimov V Kh and Roth J 2007 Phys. Scr. T 1286

[11] Kreter A et al to be submitted

[12] Doerner R P et al 2004 Phys. Scr. T 11175

[13] Nishijima D et al 2007 Phys. Plasmas 14103509

[14] Pugno R et al 2008 J. Nucl. Mater. 375168

[15] Kreter A et al 2008 J. Physics: Conf. Series 100062024

[16] Roth J et al 2007 J. Nucl. Mater. 363-365 822

[17] Baldwin M J et al 2006 J. Nucl. Mater. 35896

[18] Baldwin M J and Doerner R P 2006 Nucl. Fusion 46444

[19] Nishijima D et al 2007 J. Nucl. Mater. 363-365 1261

[20] Roth J et al 2008 Plasma Phys. Control. Fusion 50103001

[21] De Temmerman G and Doerner R P 2009 J. Nucl. Mater. 389479 